\title{
Transcriptional profiles of Burkholderia pseudomallei reveal the direct and indirect roles of Sigma E under oxidative stress conditions
}

\author{
Siroj Jitprasutwit ${ }^{1}$, Catherine $\mathrm{Ong}^{2}$, Niramol Juntawieng ${ }^{1}$, Wen Fong Ooi ${ }^{3}$, Claudia M Hemsley ${ }^{4}$, \\ Paiboon Vattanaviboon ${ }^{5}$, Richard W Titball ${ }^{4}$, Patrick $\operatorname{Tan}^{2}$ and Sunee Korbsrisate ${ }^{1^{*}}$
}

\begin{abstract}
Background: Burkholderia pseudomallei, the causative agent of melioidosis, is a Gram-negative bacterium widely distributed in soil and water in endemic areas. This soil saprophyte can survive harsh environmental conditions, even in soils where herbicides (containing superoxide generators) are abundant. Sigma factor $E\left(\sigma^{\mathrm{E}}\right)$ is a key regulator of extra-cytoplasmic stress response in Gram-negative bacteria. In this study, we identified the B. pseudomallei $\sigma^{E}$ regulon and characterized the indirect role that $\sigma^{E}$ plays in the regulation of spermidine, contributing to the successful survival of $B$. pseudomallei in stressful environments.

Results: Changes in the global transcriptional profiles of B. pseudomallei wild type and $\sigma^{\mathrm{E}}$ mutant under physiological and oxidative stress (hydrogen peroxide) conditions were determined. We identified 307 up-regulated genes under oxidative stress condition. Comparison of the transcriptional profiles of $B$. pseudomallei wild type and $\sigma^{E}$ mutant under control or oxidative stress conditions identified 85 oxidative-responsive genes regulated by $\sigma^{\mathrm{E}}$,

including genes involved in cell membrane repair, maintenance of protein folding and oxidative stress response and potential virulence factors such as a type VI secretion system (T6SS). Importantly, we identified that the speG gene, encoding spermidine-acetyltransferase, is a novel member of the $B$. pseudomallei $\sigma^{E}$ regulon. The expression of spe $G$ was regulated by $\sigma^{E}$, implying that $\sigma^{E}$ plays an indirect role in the regulation of physiological level of spermidine to protect the bacteria during oxidative stress.

Conclusion: This study identified B. pseudomallei genes directly regulated by $\sigma^{\mathrm{E}}$ in response to oxidative stress and revealed the indirect role of $\sigma^{\mathrm{E}}$ in the regulation of the polyamine spermidine (via regulation of speG) for bacterial cell protection during oxidative stress. This study provides new insights into the regulatory mechanisms by which $\sigma^{E}$ contributes to the survival of $B$. pseudomallei under stressful conditions.
\end{abstract}

Keywords: B. pseudomallei, Transcription profile, Sigma E, SpeG, Oxidative stress

\section{Background}

Burkholderia pseudomallei is a Gram-negative bacterium and the causative agent of melioidosis [1,2]. This serious and often fatal disease of humans and animals such as horses, sheep, goats, pigs and cows is endemic in Southeast Asia and northern Australia $[1,3]$. B. pseudomallei is intrinsically resistant to several antibiotics and treatment typically involves an initial parenteral phase of therapy,

\footnotetext{
*Correspondence: Sunee.kor@mahidol.ac.th

'Department of Immunology, Faculty of Medicine Siriraj Hospital, Mahidol University, Bangkok 10700, Thailand

Full list of author information is available at the end of the article
}

followed by a prolonged course of oral antibiotics [4]. No melioidosis vaccine is currently available. In endemic areas, B. pseudomallei can be found in soil and in stagnant waters [5]. In the natural environment, this saprophytic bacterium is able to survive a wide range of conditions, including fluctuating temperatures, $\mathrm{pH}$ levels, oxygen levels, osmotic pressures and nutritional stresses. During infection, the bacterium is able to survive and replicate in phagocytic or non-phagocytic cells. Within these cells B. pseudomallei may be exposed to free radicals, reactive oxygen intermediates and high osmolality. To survive exposure to stressful environments, 
B. pseudomallei must be able to activate the appropriate genes and regulate their expression. Many of these genes are organized into regulons which are under the control of sigma factors.

$\operatorname{RpoE}\left(\sigma^{\mathrm{E}}\right)$ is a member of the extra-cytoplasmic function (ECF) subfamily of sigma factors [6] and has been characterized to be one of the most important gene regulatory systems in response to extracellular stress in Gram-negative bacteria. In Escherichia coli K12, the inhibition of $\sigma^{\mathrm{E}}$ resulted in increased sensitivity to bacterial cell wall disruption [7] and in Vibrio vulnificus, deletion of $\sigma^{\mathrm{E}}$ resulted in increased sensitivity to membrane-perturbing agents such as ethanol, peroxide and SDS [8]. Inactivation of $\sigma^{\mathrm{E}}$ in Salmonella enterica serovar Typhimurium ( $S$. Typhimurium) resulted in attenuation in a mouse model of infection $[9,10]$. In addition, microarray analysis of a $S$. Typhimurium $\sigma^{\mathrm{E}}$ mutant identified the $\sigma^{\mathrm{E}}$ regulon and virulence factors that contributed to disease $[11,12]$.

A B. pseudomallei rpoE insertional inactivation mutant has previously been constructed and showed increased susceptibility to hydrogen peroxide $\left(\mathrm{H}_{2} \mathrm{O}_{2}\right)$, suggesting a role for $\sigma^{\mathrm{E}}$ in the oxidative stress response [13]. Furthermore, inactivation of $B$. pseudomallei $\sigma^{\mathrm{E}}$ resulted in reduced survival in J774A.1 macrophages and the mutant was attenuated in a murine model of infection [13,14]. A proteomic comparison of $B$. pseudomallei wild type and the $\sigma^{\mathrm{E}}$ insertional mutant revealed the differential levels of proteins that may contribute to the stress tolerance and survival of B. pseudomallei [14] but this study was unable to identify all the proteins involved in this response, because of the limitations of the proteomic platform. Recently, the development of a tiling microarray for B. pseudomallei enabled comprehensive transcriptional profiling, providing global snapshots of regulons in response to various stimuli. Analyses of transcriptional profiles of $\sigma^{\mathrm{E}}$ would lead to a better understanding of the mechanisms that bacteria use to circumvent environmental stresses. Such microarray studies will also complement our previous proteomic data and is likely to provide new insights to gene members and regulation of these genes under stress.

In this study, global transcriptional profiles of $B$. pseudomallei in response to $\mathrm{H}_{2} \mathrm{O}_{2}$-induced oxidative stress were analyzed. We compared the transcriptional profiles of B. pseudomallei wild type and its isogenic $\sigma^{\mathrm{E}}$ mutant under oxidative stress. In addition, the transcriptional profiles also revealed a novel gene member of the $\sigma^{\mathrm{E}}$ regulon, speG, that is involved in maintaining the physiological balance of the polyamine spermidine in bacterial cells during oxidative stress. This is the first report to demonstrate the direct and indirect roles of $\sigma^{\mathrm{E}}$ contributing to $B$. pseudomallei survival in the environment.

\section{Results and discussion}

\section{Comparative transcriptional profiles of $B$. pseudomallei} wild type with and without oxidative stress

The transcriptional profiles of $B$. pseudomallei in the presence or absence of $100 \mu \mathrm{M} \mathrm{H}_{2} \mathrm{O}_{2}$ for 10 min were first determined. Analyses of the profiles revealed a total of 649 genes (Additional file 1) that were differentially regulated ( $\geq 1$ absolute log-transformed fold change) representing approximately $11.0 \%$ of all $B$. pseudomallei K96243 genes. These differentially regulated genes were found on chromosome 1 (57.5\%) and chromosome 2 (42.5\%). Among the 649 genes, 307 genes were up regulated $(47.3 \%$ of differentially regulated genes) and 342 genes $(52.7 \%)$ were down regulated under oxidative stress. Since the objective of this study was to identify $B$. pseudomallei gene expression in response to oxidative stress, we focused on the analysis of the up-regulated genes. Among 307 up-regulated genes, 221 (72.0\%) could be classified into 4 major functional groups according to the Cluster of Orthologous Groups of proteins (COGs) database. These included genes involved in cell wall/ membrane biosynthesis; energy and metabolism; regulatory, signal transduction and post-translational modification; intracellular trafficking/secretion system. The remaining 86 genes $(28.0 \%)$ had unknown functions (Figure 1A).

Of the 307 up-regulated genes, 20 genes (6.5\%) were involved in cell wall/cell membrane biosynthesis (Figure 1A) including bpsl0497 (encoding periplasmic TonB protein), bpsl0785 (encoding a peptidase), bpsl3312 (encoding a putative glycosyltransferase), bpss0238 (encoding a penicillin-binding protein) and bpss0711 (encoding an alanine racemase). We also found that genes involved in the transport of lipopolysaccharide (bpsl0963) and capsular polysaccharide (bpsl2806) across the cell membrane to the bacterial cell surface were up-regulated. We found increased expression of $m r e B$ (bpsl0186) gene after oxidative stress. MreB is a bacterial ortholog of actin and MreB is reported to be important in maintaining the shape of bacteria $[15,16]$. MreB is thought to organize the incorporation of cell wall precursors into the side-wall [17]. The up-regulation of genes involved in cell wall/cell membrane synthesis may reflect their roles in the repair of the cell wall after oxidative stress damage.

In addition to genes involved in cell wall and membrane biosynthesis, 87 genes involved in energy production and metabolism were up-regulated (31\% of all up-regulated genes). These included three genes belonging to the sugar transporter superfamily (bpsl1045, bpsl2729, and bpsl2736), and two genes of the EntnerDoudoroff pathway (bpsl2931 and bpsl2932). In addition, many genes related to amino acid utilization (bpsl1076, bpsl2305 and bpsl2497) and amino acid biosynthesis (bpsl3419) were up-regulated when B. pseudomallei was 


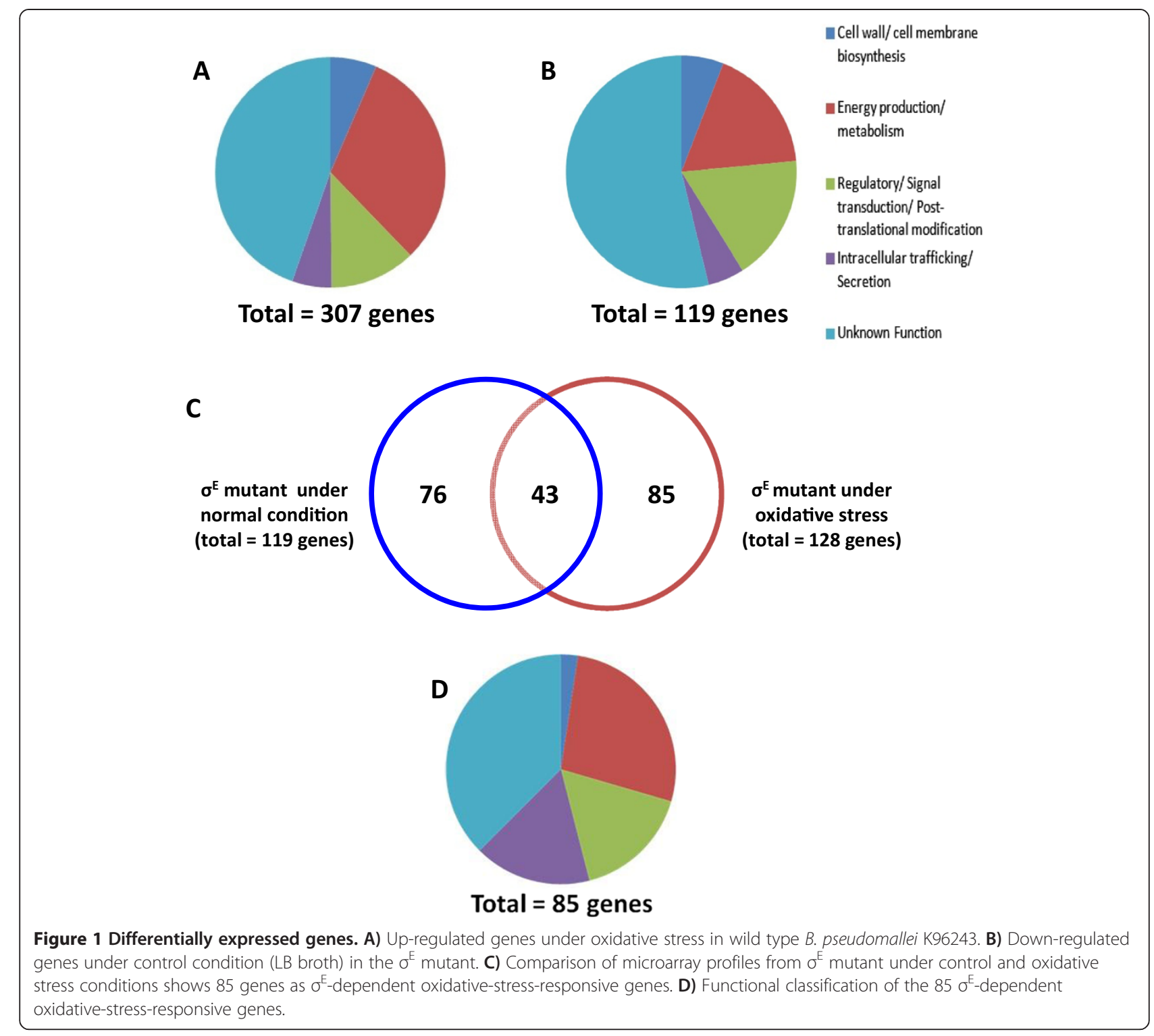

exposed to oxidative stress. We found an increased expression of B. pseudomallei bpsl1784 (encoding ATPbinding cassette transporters) which plays a role in inorganic iron transport. A previous study [18] reported that the bioavailability of iron decreases under oxidative stress conditions, and the up-regulation of bpsl1784 is consistent with this observation. The increased expression of B. pseudomallei fis-regulatory gene (bpsl0609) suggests that BPSL0609 interacts with $\sigma^{54}$ (a nitrogen specific sigma factor), with the consequential transcription of genes involved in the metabolism and transportation of nitrogen and carbon and genes involved in alginate and flagella synthesis $[19,20]$.

We observed an increase in the expression of peroxide scavenging enzymes including katG (catalase-peroxidase; bpsl2865), katB (monofunctional catalase; bpss0993) and ahpC (alkyl hydroperoxide reductase; bpss0492) during exposure to $\mathrm{H}_{2} \mathrm{O}_{2}$-induced oxidative stress (Table 1). The expression of B. pseudomallei katG and $a h p C$ is regulated through a global $\mathrm{H}_{2} \mathrm{O}_{2}$ sensor and the OxyR transcriptional regulator [21-23]. The increased expression of $k a t G$ and $a h p C$ after exposure to oxidative stress is consistent with the findings from previous studies $[22,23]$. The role of KatG may be to enable B. pseudomallei survival within phagocytes through the detoxification of antibacterial reactive oxygen species.

The differential transcription profile of B. pseudomallei under oxidative stress revealed that 37 genes $(12.1 \%$ of oxidative stress responsive genes) were predicted to encode regulatory, signal transduction or post-translational 
Table 1 Selected differentially regulated genes of $B$. pseudomallei $\mathrm{K} 96243$ and $\sigma^{\mathrm{E}}$ mutant under $\mathrm{H}_{2} \mathrm{O}_{2}$-induced oxidative stress

\begin{tabular}{|c|c|c|c|}
\hline Gene loci & Description of gene product & $\begin{array}{l}\text { Fold change: wild type under oxidative stress } \\
\text { compared with untreated control }\end{array}$ & $\begin{array}{l}\text { Fold change: } \sigma^{\mathrm{E}} \text { mutant compared } \\
\text { with wild type under oxidative stress }\end{array}$ \\
\hline \multicolumn{4}{|c|}{ Oxidative stress responsive gene (OSR gene) } \\
\hline bpss0993 & KatB & 5.90 & NS \\
\hline bpss0492 & AhpC & 4.54 & NS \\
\hline bps/2865 & KatG & 3.64 & NS \\
\hline bpss0238 & Penicillin-binding protein & 3.03 & NS \\
\hline bpss0281 & $\begin{array}{l}4 \text { Aminobutyrate } \\
\text { aminotransferase }\end{array}$ & 2.80 & NS \\
\hline bpss0711 & Alanine racemase & 2.25 & NS \\
\hline bps/3142 & BolA-like protein & 1.94 & NS \\
\hline bps12285 & Chaperone protein HscA & 1.88 & NS \\
\hline bps10497 & Periplasmic TonB protein & 1.54 & NS \\
\hline bps12286 & Co-chaperone $\mathrm{HscB}$ & 1.43 & NS \\
\hline bps/1787 & ECF sigma factors & 1.41 & NS \\
\hline bps10785 & Peptidase & 1.19 & NS \\
\hline bps10963 & Putative permease protein & 1.13 & NS \\
\hline bps12806 & Capsular polysaccharide & 1.07 & NS \\
\hline bpss0585 & $\begin{array}{l}\text { AraC family transcriptional } \\
\text { regulator }\end{array}$ & 1.07 & NS \\
\hline bps/1527 & Tex transcriptional factor & 1.05 & NS \\
\hline bps10186 & MreB & 1.04 & NS \\
\hline bps/3312 & Putative glycosyltransferase & 1.00 & NS \\
\hline \multicolumn{4}{|c|}{$\sigma^{\mathrm{E}}$-dependent and OSR gene } \\
\hline bpss 1837 & Hypothetical protein & 3.37 & -1.84 \\
\hline bpss 1839 & $\begin{array}{l}\text { Oxidative stress related } \\
\text { rubrerythrin protein }\end{array}$ & 3.36 & -1.68 \\
\hline bpss 1838 & Ferredoxin & 3.31 & -1.79 \\
\hline bps/2931 & Eda (KHG/KDPG_aldolase) & 3.26 & -2.59 \\
\hline bps/2932 & Phosphogluconate dehydratase & 3.17 & -2.13 \\
\hline bps/2605 & $\operatorname{TrxB}$ & 2.75 & -1.18 \\
\hline bps/1799 & Putative fimbrial chaperone & 2.70 & -1.71 \\
\hline bps/1800 & $\begin{array}{l}\text { Putative outer membrane } \\
\text { usher protein precursor }\end{array}$ & 2.59 & -1.86 \\
\hline bpss 1437 & Lipoprotein & 2.42 & -2.27 \\
\hline bpss 1251 & $\begin{array}{l}\mathrm{N} \text {-carbamoyl-L-amino } \\
\mathrm{N} \text {-acid amidohydrolase }\end{array}$ & 2.34 & -1.01 \\
\hline bpss 1434 & $\begin{array}{l}\text { Membrane-anchored } \\
\text { cell surface protein }\end{array}$ & 2.33 & -3.52 \\
\hline bps/3419 & Putative GMC oxidoreductase & 2.27 & -1.22 \\
\hline bpss 1252 & $\begin{array}{l}\text { Inner membrane } \\
\text { transport protein }\end{array}$ & 2.20 & -1.11 \\
\hline bps 12300 & PdhB & 2.19 & -1.34 \\
\hline bps/2301 & PdhA & 1.89 & -1.68 \\
\hline \multirow[t]{3}{*}{ bpss0175-0184 } & T6SS-4 & & \\
\hline & BPSS0175 & 1.89 & -3.67 \\
\hline & BPSS0176 & 1.77 & -3.55 \\
\hline
\end{tabular}


Table 1 Selected differentially regulated genes of $B$. pseudomallei $\mathrm{K} 96243$ and $\sigma^{\mathrm{E}}$ mutant under $\mathrm{H}_{2} \mathrm{O}_{2}$-induced oxidative stress (Continued)

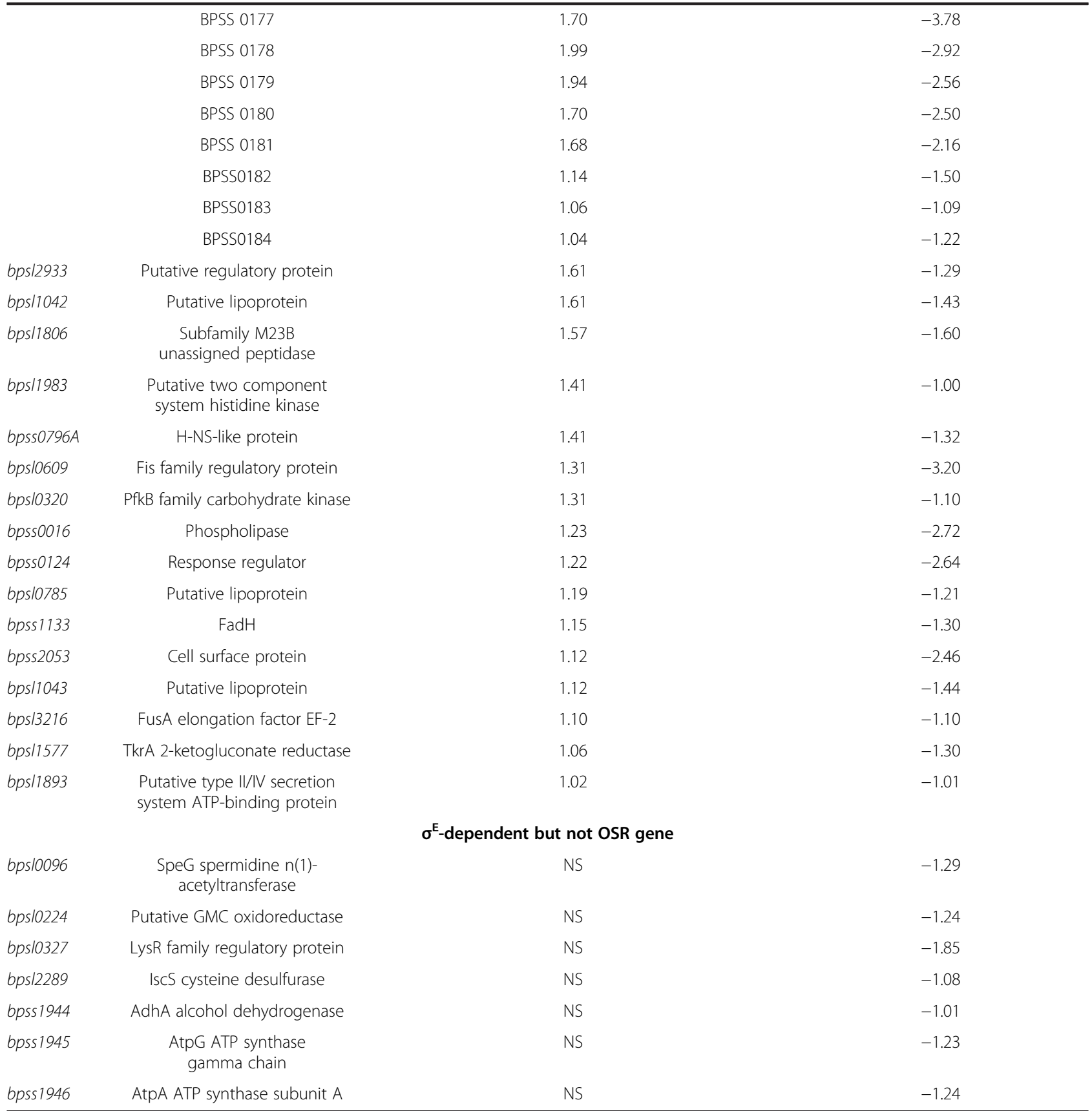

NS; Not significant different.

${ }^{*}$. p pseudomallei cultured in LB broth without $\mathrm{H}_{2} \mathrm{O}_{2}$.

modification-related proteins (Figure 1A). These genes included bpsl0049, encoding a GntR family regulatory protein and bpsl1787, encoding an ECF sigma factor. Several genes involved in transcription regulation such as tex (bpsl1527 encoding a transcriptional factor), $n r d R$ (bpsl2757 encoding a transcriptional regulator) and an $\operatorname{araC}$ family gene (bpss0585 encoding a transcriptional regulator) were upregulated. In Streptococcus pneumoniae and Pseudomonas aeruginosa the transcription factor Tex is important for bacterial fitness [24]. The NrdR transcription regulator is reported to control the expression of a ribonucleotide reductase involved in deoxyribonucleotide biosynthesis, which is required for DNA replication and repair [25]. Many members of the AraC family transcription regulator have been proven to play critical roles in regulating bacterial virulence factors in response to environmental stress 
[26]. The high number of regulatory genes up-regulated after exposure to oxidative stress may indicate that B. pseudomallei employs multiple regulation systems in response to oxidative stress.

In addition to genes involved in transcription, a number of chaperone-encoding genes were up-regulated after expsore of the bacteria to oxidative stress including hscA/hscB (bpsl2285/bpsl2286), and groES2 (bpsl2919). HscA is a specialized member of the $h s p 70$ family of molecular chaperones that plays a role in the biosynthesis of several iron-sulfur proteins [27]. Previous studies indicated the essential roles of iron-sulfur proteins in the adaptation of bacteria to iron starvation [28]. Chaperonin GroES2 binds to heat shock protein GroEL to facilitate protein folding in response to environmental stresses [29]. Oxidative stress can cause to protein misfolding, and as a result, the bacterial cells are unable to maintain their protein functions. The up-regulation of genes involved in protein folding may reflect the fact that under oxidative stress conditions, B. pseudomallei proteins are likely to become damaged.

The smallest functional group of proteins that were up-regulated under oxidative stress included 18 genes (5.5\% of total up-regulated genes) encoding proteins related to intracellular trafficking and secretion (Figure 1A). Increased expression of proteins in this group, such as type II/IV and VI secretion systems implies that the virulence of B. pseudomallei is likely to be affected by oxidative stress.

\section{Comparative analysis of transcription profiles of $B$. pseudomallei wild type and $\sigma^{\mathrm{E}}$ mutant without oxidative stress}

We have previously reported the construction of $B$. pseudomallei $\sigma^{\mathrm{E}}$ mutant. The mutant shows increased susceptibility to killing by $\mathrm{H}_{2} \mathrm{O}_{2}$, indicating the role of $\sigma^{\mathrm{E}}$ in regulating resistance to oxidative stress $[13,14]$. To identify the $\sigma^{\mathrm{E}}$ regulon under oxidative stress conditions, we first investigated the transcriptional profiles of $B$. pseudomallei wild type and the $\sigma^{\mathrm{E}}$ mutant grown in LB medium without antibiotic supplementation. Analysis of the transcription profiles revealed that a total of 350 genes (Additional file 2) were differentially regulated $(\geq 1$ absolute log fold change), representing approximately $5.9 \%$ of the total B. pseudomallei K96243 genes. These differentially regulated genes were distributed on both chromosome 1 (59.4\%) and chromosome 2 (40.6\%). In total, 231 genes were up-regulated $(66.0 \%$ of differentially regulated genes) and 119 genes (34.0\%) were downregulated in the $\sigma^{\mathrm{E}}$ mutant. The down-regulation of genes may indicate either direct or indirect regulation by $\sigma^{\mathrm{E}}$. Among the down-regulated genes, 55 (46.2\%) could be classified into 4 major COG functional groups, including 7 genes (5.9\%) predicted to be involved in cell wall/cell membrane biosynthesis, 21 genes (17.6\%) involved in energy production/metabolisms, 21 genes (17.6\%) involved in regulatory/signal transduction/post-translational modification and repair, and 6 genes (5.1\%) involved in intracellular trafficking/secretion (Figure 1B). The remaining 64 genes $(53.8 \%)$ have unknown functions.

\section{Comparative analysis of transcription profiles of $B$. pseudomallei wild type and $\sigma^{\mathrm{E}}$ mutant under oxidative stress}

To identify $\sigma^{\mathrm{E}}$-dependent genes that are differentially expressed under oxidative stress conditions, we compared the transcriptome profiles of the $\sigma^{\mathrm{E}}$ mutant and wild type which had been exposed to oxidative stress. The bacteria were treated with $\mathrm{H}_{2} \mathrm{O}_{2}$ for 10 min before RNA extraction and microarray analysis. A total of 404 genes (Additional file 3 ) were differentially regulated $(\geq 1$ absolute log fold change) representing approximately $6.81 \%$ of the total B. pseudomallei K96243 genes. Of these, 276 genes were up-regulated in the $\sigma^{\mathrm{E}}$ mutant (68.3\% of the total differentially regulated genes) and were located on either chromosome 1 (56.5\%) or chromosome 2 (43.5\%). Among the 128 down-regulated genes in the $\sigma^{\mathrm{E}}$ mutant, 43 genes were also downregulated in the mutant under normal growth conditions. By excluding these genes, we identified 85 genes defined as the $\sigma^{\mathrm{E}}$-dependent oxidative stress regulon (Figure 1C). These 85 genes were distributed on both chromosome 1 (53.1\%) and chromosome 2 (46.9\%). Two genes $(2.4 \%)$ were predicted to be involved in cell wall/ cell membrane biosynthesis, 23 genes $(27.1 \%)$ in energy production/metabolisms, 14 genes (16.5\%) in regulatory/ signal transduction/post-translational modification and repair, 14 genes (16.5\%) in intracellular trafficking/secretion. The remaining 32 genes (37.5\%) had unknown functions (Figure 1D).

Amongst the 85 genes making up the $\sigma^{\mathrm{E}}$-dependent oxidative stress regulon, bpsl1806 is predicted to be involved in cell wall/cell membrane biosynthesis and bpss0265 is predicted to encode a membrane protein related to metalloendopeptidases and porins. Genes involved in energy production and metabolism included bpsl0320-0321 (sugar kinase and N-acyl-D-glucosamine 2-epimerase), bpsl2931 (KHG/KDPG aldolase) and bpsl2300-l2301 (pyruvate dehydrogenase complex). The absence of a functional $\sigma^{\mathrm{E}}$ under oxidative stress affected the expression of bpss1838-1839 (encoding ferredoxin and rubrerythrin proteins), genes that play important roles in increasing tolerance and resistance to oxidative stress [30].

We identified $14(16.5 \%) \sigma^{\mathrm{E}}$-regulated genes involved in regulatory, signal transduction and post-translational modification after oxidative stress including bpsl0609 (encoding fis-regulatory protein), bpsl1983 (putative twocomponent system, histidine kinase), bpsl2933 (putative 
regulatory protein), bpss0124 (two-component system, response regulator) and bpsl2605 $(\operatorname{tr} x B)$. The latter encodes thioredoxin reductase which functions in posttranslational modification. In addition, site-specific recombinase (bpsl2881), which is involved in DNA replication, recombination and repair, was also under $\sigma^{\mathrm{E}}$ regulation.

Intracellular trafficking and secretion genes accounted for $12.8 \%$ of $\sigma^{\mathrm{E}}$-dependent oxidative stress responsive genes. These included genes of the type II secretion system (bpsl1893), fimbrial proteins (bpsl1798-1800) and membrane-anchored cell surface protein (bpss1434). B. pseudomallei contains six clusters type VI secretion system (T6SS-1 to T6SS-6) [31]. The expression of T6SS genes has been reported to be induced in vivo [31-33]. A previous study reported that the T6SS-1 cluster is important for host adaptation of B. pseudomallei within phagocytes, and that the expression of genes in this cluster is significantly elevated after infection of murine macrophages [31]. We found the increased expression of ten genes (bpss0175-0184) belonging to T6SS-4 under oxidative stress conditions suggesting that T6SS-4 may play a role in combating oxidative stress.

\section{RT-PCR analysis of genes under normal and oxidative stress conditions}

To validate the results from our microarray analysis, RT-PCR was performed. Figure 2 shows the increased expression of the bpsl0124 and bpss1434 genes in B. pseudomallei wild type after $\mathrm{H}_{2} \mathrm{O}_{2}$ treatment. We did not observe a significant difference in expression of bpsl0096 (speG; encoding spermidine-n-1-acetyltransferase). These results are consistent with the microarray

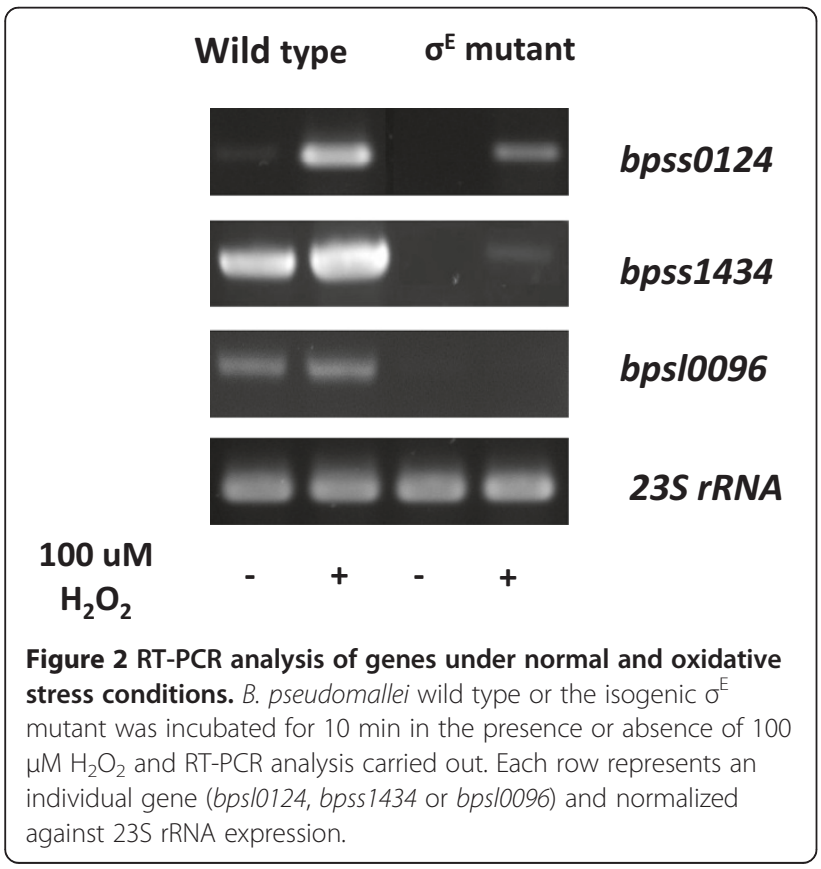

data which indicated that bpsl0124 and bpss1434, but not bpsl0096, were up-regulated in response to oxidative stress (Additional file 1). In the B. pseudomallei $\sigma^{\mathrm{E}}$ mutant exposed to $\mathrm{H}_{2} \mathrm{O}_{2}$ treatment, the expression of the bpsl0124, bpss1434 and bpsl0096 genes was down-regulated compared to the wild type, indicating that these genes are under $\sigma^{\mathrm{E}}$ control. These results are also consistent with our microarray results (Additional file 2).

In addition to RT-PCR, the differential transcription profiles were analyzed to ensure the quality of our transcription profile data. For example, we found that 10 genes (bpss0175-0184) encoding B. pseudomallei T6SS-4 were all up-regulated, suggesting that these genes may be co-expressed as an operon and support the validity of our transcriptional profiling results (Table 1).

Other studies have shown that the katG (catalase-peroxidase) and $a h p C$ (alkyl hyperoxide reductase) genes are up-regulated following the exposure of B. pseudomallei to oxidative stress [22,23]. Our transcriptional data (Table 1) also reveals these patterns of gene expression. Collectively, these results indicate that our data is robust and reliable.

\section{B. pseudomallei $\sigma^{\mathrm{E}}$ indirectly regulates spermidine levels during oxidative stress}

Previous studies have suggested that sigma factors regulate speG and consequently spermidine levels [34,35]. Spermidine is one of the predominant polyamines in Gram-negative bacteria, widely distributed in the environment, and is involved in various biological processes including gene regulation, protein translation and stress resistance [36]. During oxidative stress, spermidine functions as a free radical scavenger and plays an important adjunctive role in protecting bacterial cells from the toxic effects of reactive oxygen species [37]. The intracellular level of spermidine in bacteria is reported to range from 1-3 mM [38]. High concentrations of spermidine are toxic for bacteria. Excess spermidine can be a result of deregulated bacterial biosynthesis/metabolism or from environmental exposure, inhibiting bacterial growth and even killing the bacterial cells [39]. Therefore, in bacteria, the maintenance of an appropriate intracellular level of spermidine is critical. Excess spermidine can be converted into the physiologically inert acetylspermidine by the spermidine-acetyltransferase (SpeG). A recent study revealed that the speG gene has been silenced by convergent evolution in Shigella and this resulted in elevated levesl of intracellular spermidine. As a result, the survival of Shigella under oxidative stress is enhanced, contributing to its successful pathogenic lifestyle [40].

We observed expression of speG (bpsl0096) in $B$. pseudomallei wild type under both control and oxidative stress conditions (Figure 2). However, the speG gene was down-regulated in the $\sigma^{\mathrm{E}}$ mutant (Additional file 3), 
indicating that the inactivation of $r p o E$ effected the expression of speG. This suggests that speG is regulated by $\sigma^{E}$. The decreased gene expression we have observed corroborates our previous proteomic study [14]. To our knowledge, this is the first report on the regulation of spe $G$ by $\sigma^{\mathrm{E}}$.

We hypothesised that under high concentrations of spermidine, $\sigma^{\mathrm{E}}$ positively regulate expression of speG gene in order to prevent spermidine accumulation, the failure of which, will result in inhibition of $B$. pseudomallei growth and even cytotoxicity. To test this hypothesis, cultures of B. pseudomallei wild type or $\sigma^{\mathrm{E}}$ mutant grown in LB broth were exposed to $1 \mathrm{mM}$ spermidine and the number of viable bacteria determined. The number of wild type bacteria was not affected by the addition of spermidine. This is likely due to the presence of a functional $\sigma^{\mathrm{E}}$ gene in the wild type, activating the expression of speG gene. In contrast, in the B. pseudomallei $\sigma^{\mathrm{E}} \mathrm{mu}-$ tant, the number of viable bacteria was significantly reduced by the addition of spermidine, indicating the accumulation of spermidine to toxic levels (Figure 3 ).

This result corroborates the findings from a previous study in $E$. coli, where excess spermidine was shown to be toxic to bacteria and speG was shown to be important for bacterial viability [41]. Our study provides further evidence that the regulation of speG is affected by $\sigma^{\mathrm{E}}$; spe $G$ is a novel member of the $\sigma^{\mathrm{E}}$ regulon and $\sigma^{\mathrm{E}}$ plays an indirect but important role in the regulation of polyamine levels in bacterial cells to protect the cells during oxidative stress.

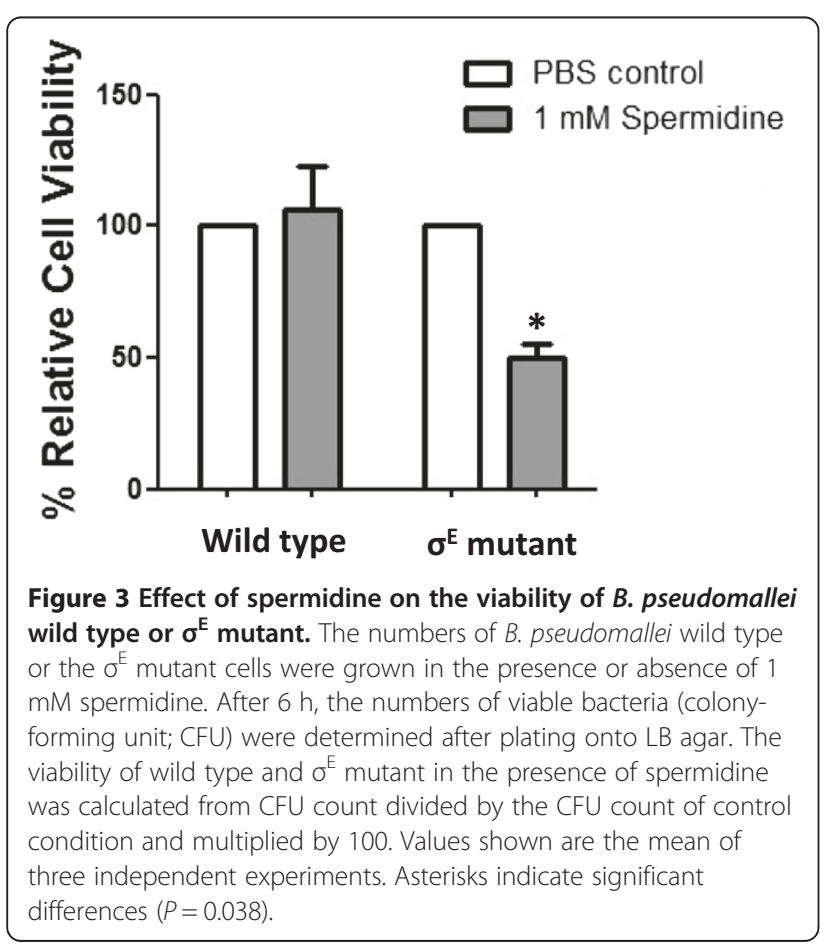

\section{Conclusions}

DNA tiling arrays were employed to identify global transcriptional profile changes in B. pseudomallei K96243 exposed to oxidative stress induced by $\mathrm{H}_{2} \mathrm{O}_{2}$. We have identified not only genes involved in repairing cell wall/ membrane biosynthesis but also genes involved in energy and metabolism, regulatory and signal transduction, post translational modification, and intracellular trafficking/secretion genes, which are directly regulated by $\sigma^{\mathrm{E}}$ during oxidative stress. We found the increased expression of the B. pseudomallei T6SS-4 under oxidative stress. More importantly, we provided evidence that $\sigma^{\mathrm{E}}$ also indirectly regulates the polyamine levels in B. pseudomallei, to protect the cells from oxidative stress.

\section{Methods}

\section{Bacterial strains and culture conditions}

B. pseudomallei $\mathrm{K} 96243$ wild type or the $\sigma^{\mathrm{E}}$ mutant [13] was grown at $37^{\circ} \mathrm{C}$ in Luria-Bertani (LB) broth or $\mathrm{LB}$ agar (Criterion) with or without $50 \mu \mathrm{g} / \mathrm{ml}$ of chloramphenicol (Sigma).

\section{Extraction of bacterial total RNA}

B. pseudomallei wild type K96243 or the isogenic $\sigma^{\mathrm{E}} \mathrm{mu}$ tant was harvested after culturing in LB broth without chloramphenicol supplementation. After centrifugation, the cell pellet was washed and treated with TRIZOL (Invitrogen). One-tenth volume of 1-bromo-3-chloropropane (Sigma) was added to the mixture before centrifugation. The aqueous phase was transferred to a fresh tube containing equal volume of isopropanol to precipitate the total RNA. After centrifugation, the supernatant was discarded; the RNA pellet was washed with $75 \%$ ethanol and suspended in RNase-free water. RNA concentration was quantified by spectrophotometer. The isolated bacterial RNA was then treated with DNase I (Ambion) according to manufacturer's instructions to remove any genomic DNA contamination. DNase inactivation reagent (Ambion) was then added to stop the reaction.

\section{Bacterial mRNA enrichment, cDNA synthesis and microarray hybridization}

Bacterial mRNA was enriched from purified total RNA and synthesized into single-stranded cDNA prior to microarray hybridization as described in [42]. The purified cDNAs prepared from B. pseudomallei wild type or $\sigma^{\mathrm{E}}$ mutant were labeled with $\mathrm{Cy} 5$ or $\mathrm{Cy} 3$ respectively (Cy5-ULS Cy3-ULS, Kreatech Diagnostics). Hybridization of labeled cDNA to the array was performed and images acquired from array slides as previously described [43]. Data obtained from hybridizations of two independent RNA preparations of each bacterial strain were used in each analysis. 


\section{Design of B. pseudomallei K96243 high-density tiling microarray}

A high-density tiling array based on the sequenced reference genome B. pseudomallei K96243 was customfabricated using NimbleGen's photolithographic Maskless Array Synthesis (MAS) platform (Roche NimbleGen). Using the 7.2 Mb B. pseudomallei K96243 genome sequence, we selected 384,926 50 mer oligonucleotide probes to represent both sense and antisense strands of the $B$. pseudomallei genome at an average resolution of $35 \mathrm{bp}$ (probes have a mean overlap of $15 \mathrm{bp}$ ). Control features that are not complementary to B. pseudomallei K96243 genome, were also included for background checks and alignment purposes. Altogether, $95.1 \%$ of the B. pseudomallei K96243 genome, including intergenic regions, is represented on this high-density tiling array.

\section{Data acquisition and preprocessing}

Images were acquired with Axon GenePix 4000B laser scanner (Molecular Devices) at $5 \mu \mathrm{m}$ resolution and intensity data were extracted using the software NimbleScan (Roche NimbleGen). Data obtained from hybridizations of two independent RNA preparations of each sample were used for final analysis. Raw microarray data were first LOWESS (Locally Weighted Scatter Plot Smoother) normalized using GeneSpring GX (Agilent) to correct for dye-bias within array followed by median normalization to normalize across all arrays. Finally, the median ratio of probes corresponding to Sanger's 5935 genes comparing between $B$. pseudomallei wild type and $\sigma^{\mathrm{E}}$ mutant was computed.

\section{Differential expression analysis}

Changes in the expression of genes under oxidative stress (denoted as $\mathrm{T}$ ) compared to control conditions (denoted as R) were measured in $\log _{2}$ fold change [44]. Specifically, each condition was normalized by a common reference, of which intensity was measured in arrays with Cy5 channels. The common reference $\left(\mathrm{R}^{\mathrm{C}}\right)$ is B. pseudomallei $\mathrm{K} 96243$ grown to stationary phase in LB broth. We computed the difference of two normalized values as the log-transformed fold change: $\log (T / R)=\log$ $\left(T / R^{c}\right)-\log \left(R / R^{c}\right)$. Genes with a $\log _{2}$ fold change $\geq 1$ were considered further. The microarray data have been deposited in Gene Expression Omnibus (GEO) with the identifier, GSE43205. In particular, the data used in this study are as follow: GSM1058304 (sigmaE-mutant + oxidative stress), GSM1058305 (common reference for sigmaE-mutant + oxidative stress), GSM1058306 (sigmaE-mutant control), GSM1058307 (common reference for sigmaE-mutant control), GSM1058508 (wild type + oxidative stress), GSM1058509 (common reference for wild type + oxidative stress), GSM1058519 (wild type control), GSM1058520 (common reference for wild type control).

\section{RT-PCR analysis}

An overnight cultured of B. pseudomallei was subcultured in $10 \mathrm{ml}$ of $\mathrm{LB}$ broth before incubation at $37^{\circ} \mathrm{C}$ for $6 \mathrm{~h}\left(\mathrm{OD}_{600}\right.$ of 0.8$)$. The logarithmic phase cells were centrifuged and washed with $1 \mathrm{x}$ PBS and resuspended into $10 \mathrm{ml}$ of $\mathrm{LB}$ broth containing $100 \mu \mathrm{M} \mathrm{H} \mathrm{H}_{2} \mathrm{O}_{2}$ before incubation at $37^{\circ} \mathrm{C}$ for $10 \mathrm{~min}$. After $\mathrm{H}_{2} \mathrm{O}_{2}$ treatment, bacterial RNA was extracted using Total RNA mini Kit (GeneAid) according to the manufacturer's protocol. To remove trace genomic DNA, the RNA samples were treated with DNase I (Promaga). The yield and purity of the RNA were determined by spectrophotometer (Nanodrop Technologies). The absence of DNA contamination was confirmed by PCR before proceeding to cDNA synthesis.

SuperScript III First-Strand Synthesis System (Invitrogen) was used to convert total RNA to cDNA. The cDNA was amplified using the PCR with primers (Table 2), GoTaq DNA polymerase (Promega) and cycling conditions of $94^{\circ} \mathrm{C}, 3 \mathrm{~min}$ and 30 cycles of $94^{\circ} \mathrm{C}$ for $30 \mathrm{~s}, 55^{\circ} \mathrm{C}$ for $1 \mathrm{~min}$, and $72^{\circ} \mathrm{C}$ for $45 \mathrm{~s}$, followed by incubation at $72^{\circ} \mathrm{C}$ for $5 \mathrm{~min}$. In each PCR experiment, the amplification of $23 \mathrm{~S}$ rRNA was used as a normalization control. The amplified products were then visualized using GeneSys software (Syngene). Positive controls were performed with genomic DNA, and negative controls were performed with RNA that had not been subjected to reverse transcription.

\section{Spermidine sensitivity assay}

The numbers of $B$. pseudomallei wild type or the $\sigma^{\mathrm{E}} \mathrm{mu}-$ tant cells were adjusted to $100 \mathrm{CFU}$ and subjected to grow in the presence or absence of $1 \mathrm{mM}$ spermidine (Sigma). After $6 \mathrm{~h}$, the bacterial samples were plated onto LB agar to determine the numbers of viable bacteria as CFU. The cell viability of $B$. pseudomallei under control condition was set as $100 \%$. The viability of $B$. pseudomallei in the presence of spermidine was

Table 2 Oligonucleotide primers used in this study

\begin{tabular}{lll}
\hline Primer sequence $\left(\mathbf{5}^{\prime} \rightarrow \mathbf{3}^{\prime}\right)$ & Purpose \\
\hline 23S-F & TाTCCCGCTAG ATG CTT & Forward primer for 23S rRNA \\
23S-R & AAAGGTACTCTGGGGATAA & Reverse primer for 23S rRNA \\
bps/0096-F & TCGATTAGTTCGGCCTCGTG & Forward primer for bps/0096 \\
bps/0096-R & GAGCTCGACTACATCCACCG & Reverse primer for bps/0096 \\
bps/0124-F & ATTATGACGAATGGGAGCAG & Forward primer for bps/0124 \\
bps10124-R & GCGCTTGTTGATGATGAAAT & Reverse primer for bps/0124 \\
bpss1434-F & GTCGAAGGACGTGAACAGTG & Forward primer for bpss1434 \\
bpss1434-R & ACACGAGAAATTCGGACAC & Reverse primer for bpss1434 \\
\hline
\end{tabular}


calculated from CFU count in the presence of spermidine divided by the CFU count of control condition and multiplied by 100 .

\section{Statistical analysis}

Average and standard errors of the mean (SEM) were calculated from at least three independent determinations. All tests for significance were performed using the Student's $t$-test. A $P$-value $<0.05$ was considered statistically significant.

\section{Additional files}

\section{Additional file 1: Differentially expressed gene of $B$. pseudomallei $\mathrm{K} 96243$ under $\mathrm{H}_{2} \mathrm{O}_{2}$-induced oxidative stress. \\ Additional file 2: Differentially expressed gene of $B$. pseudomallei $\sigma^{\mathrm{E}}$ mutant and $\mathrm{K} 96243$ wild type under physiological condition. \\ Additional file 3: Differentially expressed gene of B. pseudomallei $\sigma^{\mathrm{E}}$ mutant and K96243 wild type under oxidative stress.}

\section{Competing interests}

The authors declare that have no competing interests.

\section{Authors' contributions}

CO and WO performed microarray hybridization and analysis. SJ and SK prepared B. pseudomallei RNA, performed bacterial functional assays. SJ, CO, RT and SK wrote the manuscript. SJ and NJ performed RT-PCR. CH, PT, PV, RT and SK participated in study design, coordination or extensive revised the manuscript. All authors read and approved the final manuscript.

\section{Acknowledgements}

This work was supported by the National Science and Technology Development Agency and Siriraj Grant for Research and Development. S. Jitprasutwit was supported by the Royal Golden Jubilee Ph. D. Program (PHD0270/2551)

\section{Author details}

${ }^{1}$ Department of Immunology, Faculty of Medicine Siriraj Hospital, Mahidol University, Bangkok 10700, Thailand. 'DSO National Laboratories, Singapore 117510, Singapore. ${ }^{3}$ Genome Institute of Singapore, Singapore 138672 , Singapore. ${ }^{4}$ Bioscience, Geoffrey Pope Building, University of Exeter, Devon EX4 4QD, UK. '5aboratory of Biotechnology, Chulabhorn Research Institute, Bangkok 10210, Thailand.

Received: 29 March 2014 Accepted: 8 September 2014 Published: 12 September 2014

\section{References}

1. Abraham KA: Studies on DNA-dependent RNA polymerase from Escherichia coli. 1. The mechanism of polyamine induced stimulation of enzyme activity. Eur J Biochem 1968, 5(1):143-146.

2. Wuthiekanun V, Mayxay M, Chierakul W, Phetsouvanh R, Cheng AC, White NJ, Day NP, Peacock SJ: Detection of Burkholderia pseudomallei in soil within the Lao People's Democratic Republic. J Clin Microbiol 2005, 43(2):923-924.

3. Wiersinga WJ, van der Poll T, White NJ, Day NP, Peacock SJ: Melioidosis: insights into the pathogenicity of Burkholderia pseudomallei. Nat Rev Microbiol 2006, 4(4):272-282.

4. Kanthawong S, Nazmi K, Wongratanacheewin S, Bolscher JG, Wuthiekanun V, Taweechaisupapong S: In vitro susceptibility of Burkholderia pseudomallei to antimicrobial peptides. Int J Antimicrob Agents 2009, 34(4):309-314.

5. Wuthiekanun V, Smith MD, White NJ: Survival of Burkholderia pseudomallei in the absence of nutrients. Trans R Soc Trop Med Hyg 1995, 89(5):491.

6. De Las PA, Connolly L, Gross CA: SigmaE is an essential sigma factor in Escherichia coli. J Bacteriol 1997, 179(21):6862-6864.
7. Hayden JD, Ades SE: The extracytoplasmic stress factor, sigmaE, is required to maintain cell envelope integrity in Escherichia coli. PLoS One 2008, 3(2):e1573.

8. Brown RN, Gulig PA: Roles of RseB, sigmaE, and DegP in virulence and phase variation of colony morphotype of Vibrio vulnificus. Infect Immun 2009, 77(9):3768-3781.

9. Humphreys S, Stevenson A, Bacon A, Weinhardt AB, Roberts M: The alternative sigma factor, sigmaE, is critically important for the virulence of Salmonella typhimurium. Infect Immun 1999, 67(4):1560-1568.

10. Testerman TL, Vazquez-Torres A, Xu Y, Jones-Carson J, Libby SJ, Fang FC: The alternative sigma factor sigmaE controls antioxidant defences required for Salmonella virulence and stationary-phase survival. Mol Microbiol 2002, 43(3):771-782

11. Skovierova H, Rowley G, Rezuchova B, Homerova D, Lewis C, Roberts M, Kormanec J: Identification of the sigmaE regulon of Salmonella enterica serovar Typhimurium. Microbiology 2006, 152(Pt 5):1347-1359.

12. Lewis C, Skovierova H, Rowley G, Rezuchova B, Homerova D, Stevenson A, Spencer J, Farn J, Kormanec J, Roberts M: Salmonella enterica Serovar Typhimurium HtrA: regulation of expression and role of the chaperone and protease activities during infection. Microbiology 2009, 155(Pt 3):873-881.

13. Korbsrisate S, Vanaporn M, Kerdsuk P, Kespichayawattana W, Vattanaviboon P, Kiatpapan P, Lertmemongkolchai G: The Burkholderia pseudomallei RpoE $(\mathrm{AlgU})$ operon is involved in environmental stress tolerance and biofilm formation. FEMS Microbiol Lett 2005, 252(2):243-249.

14. Thongboonkerd V, Vanaporn M, Songtawee N, Kanlaya R, Sinchaikul S, Chen ST, Easton A, Chu K, Bancroft GJ, Korbsrisate S: Altered proteome in Burkholderia pseudomallei rpoE operon knockout mutant: insights into mechanisms of rpoE operon in stress tolerance, survival, and virulence. J Proteome Res 2007, 6(4):1334-1341.

15. Figge RM, Divakaruni AV, Gober JW: MreB, the cell shape-determining bacterial actin homologue, co-ordinates cell wall morphogenesis in Caulobacter crescentus. Mol Microbiol 2004, 51(5):1321-1332.

16. Doi M, Wachi M, Ishino F, Tomioka S, Ito M, Sakagami Y, Suzuki A, Matsuhashi $M$ : Determinations of the DNA sequence of the $m r e B$ gene and of the gene products of the mre region that function in formation of the rod shape of Escherichia coli cells. J Bacterio/ 1988, 170(10):4619-4624.

17. Gaballah A, Kloeckner A, Otten C, Sahl HG, Henrichfreise B: Functional analysis of the cytoskeleton protein MreB from Chlamydophila pneumoniae. PLoS One 2011, 6(10):e25129.

18. Pantopoulos K, Hentze MW: Rapid responses to oxidative stress mediated by iron regulatory protein. EMBO J 1995, 14(12):2917-2924.

19. Cases I, Ussery DW, de Lorenzo V: The sigma54 regulon (sigmulon) of Pseudomonas putida. Environ Microbiol 2003, 5(12):1281-1293.

20. Studholme DJ, Wigneshwereraraj SR, Gallegos MT, Buck M: Functionality of purified sigma(N) (sigma(54)) and a NifA-like protein from the hyperthermophile Aquifex aeolicus. J Bacterio/ 2000, 182(6):1616-1623.

21. Dowling AJ, Wilkinson PA, Holden MT, Quail MA, Bentley SD, Reger J, Waterfield NR, Titball RW, Ffrench-Constant RH: Genome-wide analysis reveals loci encoding anti-macrophage factors in the human pathogen Burkholderia pseudomallei K96243. PLoS One 2010, 5(12):e15693.

22. Loprasert S, Whangsuk W, Sallabhan R, Mongkolsuk S: Regulation of the $k a t G-d p s A$ operon and the importance of KatG in survival of Burkholderia pseudomallei exposed to oxidative stress. FEBS Lett 2003, 542(1-3):17-21

23. Loprasert S, Sallabhan R, Whangsuk W, Mongkolsuk S: Compensatory increase in $a h p C$ gene expression and its role in protecting Burkholderia pseudomallei against reactive nitrogen intermediates. Arch Microbiol 2003, 180(6):498-502.

24. He X, Thornton J, Carmicle-Davis S, McDaniel LS: Tex, a putative transcriptional accessory factor, is involved in pathogen fitness in Streptococcus pneumoniae. Microb Pathog 2006, 41(6):199-206.

25. Nordlund P, Reichard P: Ribonucleotide reductases. Annu Rev Biochem 2006, 75:681-706.

26. Gallegos MT, Schleif R, Bairoch A, Hofmann K, Ramos JL: Arac/XylS family of transcriptional regulators. Microbiol Mol Biol Rev 1997, 61(4):393-410.

27. Vickery LE, Cupp-Vickery JR: Molecular chaperones HscA/Ssq1 and HscB/ Jac1 and their roles in iron-sulfur protein maturation. Crit Rev Biochem Mol Biol 2007, 42(2):95-111.

28. Wandersman C, Delepelaire P: Bacterial iron sources: from siderophores to hemophores. Annu Rev Microbiol 2004, 58:611-647. 
29. Llorca O, Galan A, Carrascosa JL, Muga A, Valpuesta JM: GroEL under heat-shock. Switching from a folding to a storing function. $J$ Biol Chem 1998, 273(49):32587-32594.

30. Sztukowska M, Bugno M, Potempa J, Travis J, Kurtz DM Jr: Role of rubrerythrin in the oxidative stress response of Porphyromonas gingivalis. Mol Microbiol 2002, 44(2):479-488.

31. Shalom G, Shaw JG, Thomas MS: In vivo expression technology identifies a type VI secretion system locus in Burkholderia pseudomallei that is induced upon invasion of macrophages. Microbiology 2007, 153(Pt 8):2689-2699.

32. Das S, Chakrabortty A, Banerjee R, Roychoudhury S, Chaudhuri K: Comparison of global transcription responses allows identification of Vibrio cholerae genes differentially expressed following infection. FEMS Microbiol Lett 2000, 190(1):87-91.

33. Potvin E, Lehoux DE, Kukavica-lbrulj I, Richard KL, Sanschagrin F, Lau GW, Levesque RC: In vivo functional genomics of Pseudomonas aeruginosa for high-throughput screening of new virulence factors and antibacterial targets. Environ Microbiol 2003, 5(12):1294-1308.

34. Samuel Raj V, Full C, Yoshida M, Sakata K, Kashiwagi K, Ishihama A, Igarashi $K$ : Decrease in cell viability in an RMF, sigma(38), and OmpC triple mutant of Escherichia coli. Biochem Biophys Res Commun 2002, 299(2):252-257.

35. Terui $Y$, Higashi $K$, Tabei $Y$, Tomitori $H$, Yamamoto K, Ishihama A, Igarashi $K$, Kashiwagi K: Enhancement of the synthesis of RpoE and StpA by polyamines at the level of translation in Escherichia coli under heat shock conditions. J Bacteriol 2009, 191(17):5348-5357.

36. Shah P, Swiatlo E: A multifaceted role for polyamines in bacterial pathogens. Mol Microbiol 2008, 68(1):4-16

37. Ha HC, Sirisoma NS, Kuppusamy P, Zweier JL, Woster PM, Casero RA Jr: The natural polyamine spermine functions directly as a free radical scavenger. Proc Natl Acad Sci U S A 1998, 95(19):11140-11145.

38. Cohen SS: A guide to the polyamines. New York; Oxford: Oxford University Press; 1998.

39. He Y, Kashiwagi K, Fukuchi J, Terao K, Shirahata A, Igarashi K: Correlation between the inhibition of cell growth by accumulated polyamines and the decrease of magnesium and ATP. Eur J Biochem 1993, 217(1):89-96.

40. Barbagallo M, Di Martino ML, Marcocci L, Pietrangeli P, De Carolis E, Casalino M, Colonna B, Prosseda G: A new piece of the Shigella pathogenicity puzzle: spermidine accumulation by silencing of the speG gene. PLoS One 2011, 6(11):e27226.

41. Fukuchi J, Kashiwagi K, Yamagishi M, Ishihama A, Igarashi K: Decrease in cell viability due to the accumulation of spermidine in spermidine acetyltransferase-deficient mutant of Escherichia coli. J Biol Chem 1995, 270(32):18831-18835.

42. Sun GW, Chen Y, Liu Y, Tan GY, Ong C, Tan P, Gan YH: Identification of a regulatory cascade controlling Type III Secretion System 3 gene expression in Burkholderia pseudomallei. Mol Microbiol 2010, 76(3):677-689.

43. Ong C, Ooi CH, Wang D, Chong H, Ng KC, Rodrigues F, Lee MA, Tan P: Patterns of large-scale genomic variation in virulent and avirulent Burkholderia species. Genome Res 2004, 14(11):2295-2307.

44. Ooi WF, Ong C, Nandi T, Kreisberg JF, Chua HH, Sun G, Chen Y, Mueller C, Conejero L, Eshaghi M, Ang RM, Liu J, Sobral BW, Korsrisate S, Gen YH, Titball RW, Bancroft GJ, Valade E, Tan P: The condition-dependent transcriptional landscape of Burkholderia pseudomallei. PLoS Genet 2013, 9(9):e1003795.

doi:10.1186/1471-2164-15-787

Cite this article as: Jitprasutwit et al:: Transcriptional profiles of Burkholderia pseudomallei reveal the direct and indirect roles of Sigma E under oxidative stress conditions. BMC Genomics 2014 15:787.

\section{Submit your next manuscript to BioMed Central and take full advantage of:}

- Convenient online submission

- Thorough peer review

- No space constraints or color figure charges

- Immediate publication on acceptance

- Inclusion in PubMed, CAS, Scopus and Google Scholar

- Research which is freely available for redistribution

Submit your manuscript at www.biomedcentral.com/submit
() Biomed Central 